\title{
Construction of exact constants of motion and effective models for many-body localized systems
}

\author{
M. Goihl, M. Gluza, C. Krumnow, and J. Eisert \\ Dahlem Center for Complex Quantum Systems, Freie Universität Berlin, 14195 Berlin, Germany
}

(Received 7 August 2017; published 16 April 2018)

\begin{abstract}
One of the defining features of many-body localization is the presence of many quasilocal conserved quantities. These constants of motion constitute a cornerstone to an intuitive understanding of much of the phenomenology of many-body localized systems arising from effective Hamiltonians. They may be seen as local magnetization operators smeared out by a quasilocal unitary. However, accurately identifying such constants of motion remains a challenging problem. Current numerical constructions often capture the conserved operators only approximately, thus restricting a conclusive understanding of many-body localization. In this work, we use methods from the theory of quantum many-body systems out of equilibrium to establish an alternative approach for finding a complete set of exact constants of motion which are in addition guaranteed to represent Pauli- $z$ operators. By this we are able to construct and investigate the proposed effective Hamiltonian using exact diagonalization. Hence, our work provides an important tool expected to further boost inquiries into the breakdown of transport due to quenched disorder.
\end{abstract}

DOI: 10.1103/PhysRevB.97.134202

\section{INTRODUCTION}

The question of the precise mechanism of thermalization of closed quantum many-body systems lies at the heart of the foundations of quantum statistical mechanics. For generic systems, one generally expects that the unitary time evolution evolves the system into states that can locally be captured by a thermodynamic ensemble using only few parameters such as the total energy or particle number [1-3]. This expectation will be violated if additional structure is present in the system that enforces a local memory of initial conditions by confining, for instance, particles to local regions. Such a nonthermalizing behavior caused by localization is most famously observed due to quenched disorder in Anderson insulators [4] and prevails under the addition of interactions in the form of many-body localization (MBL) as predicted theoretically [5-7] and observed experimentally [8,9].

These systems are expected to feature extensively many quasilocal constants of motion (qLCOMs) which prevent a thermodynamic description. In stark contrast to the Anderson insulator, many-body localized systems feature a slow, unbounded growth of entanglement due to interactions $[10,11]$. Moreover, all MBL eigenstates are expected to fulfill an entanglement area law [12-14], which delineates them from the eigenstates of thermalizing systems while making them amenable to tensor network approaches [14-19]. Due to their special structure, MBL systems are candidates for understanding fundamental aspects of quantum mechanics, microscopic transport properties, and interacting systems as their efficient description appears to be in reach.

One of the most successful explanations of the intriguing behavior of MBL systems has been through a proposed effective Hamiltonian valid in the strong disorder limit, stated by employing a complete set of qLCOMs [20,21]. This description explains the logarithmic entanglement growth $[10,22,23]$. For the case of disordered spin chains the qLCOMs are considered to be dressed local magnetization operators, i.e., local spin operators conjugated by a unitary transformation smearing their support within an exponential envelope but at the same time promoting them to constants of motion. Under reasonable assumptions these operators can actually be calculated analytically for a specific MBL model [24] that is disordered in all parameters. For models which contain disorder only in the form of local potentials, much in the spirit of current experimental investigations, no analytical results are known as of today. It is hence unclear if more physical models of MBL, such as the disordered Heisenberg chain, can actually be mapped to the effective Hamiltonian of Refs. [20,21]. We report on progress in developing a machinery to numerically construct exact quasilocal constants of motion.

Among the strategies established so far are several variants of transformation schemes which focus on decoupling the Hamiltonian [25-29] and by this implicitly define qLCOMs. These approaches have the advantage of being able to treat larger systems at the cost of making specific approximations, whose exact effects need to be understood [30,31]. For small systems exact diagonalization based methods can be used [32-34]. While in general quite arbitrary operators qualify as constants of motion, one aims to ensure specific attributes when constructing them numerically. The qLCOMs are supposed to be quasilocal, resemble Pauli- $z$ operators by being traceless with only two degenerate eigenvalues, and mutually commute among each other and of course with the Hamiltonian of the system. Different numerical schemes trade these properties differently against each other. Whenever exact diagonalization is feasible then qLCOMs can be constructed directly, e.g., via optimizing the commutant matrix $[33,34]$ or performing the infinite-time average [32] which also inspired our work. The latter methods perturb the spectrum and the qLCOMs are not dressed spins anymore and the former study shows that when a local region is embedded into a larger one then the optimal qLCOMs conditioned on the subsystem size could be a superposition of several dressed spin operators because of tail cancellation. In neither of these 
exact diagonalization (ED) studies it was possible to construct the effective Hamiltonian of Refs. [20,21] in order to support the large-scale transformation schemes.

In this work we present a scheme for computing constants of motion that allows us to study the effective Hamiltonian. The idea behind our construction follows a clear physical intuition: Quasilocal conservation of the local magnetization implies that the corresponding local Pauli- $z$ operators remain approximately local under time evolution. We show numerically that infinite time-averaged magnetization operators can be promoted to true Pauli- $z$ operators, while keeping the locality properties intact and gaining the desired spectrum by construction. Our construction fails to be local if the time evolution ergodically spreads local excitations and is hence physically directly connected to the breakdown of MBL. Equipped with a full set of exact qLCOMs we go a step further and study the effective model of Refs. [20,21] for the disordered Heisenberg chain, which is based on obtained qLCOMs.

\section{SETTING}

We consider the prototypical model of MBL, the disordered spin-1/2-Heisenberg chain on $L$ sites

$$
H=\sum_{i=1}^{L}\left(\sigma_{i}^{x} \sigma_{i+1}^{x}+\sigma_{i}^{y} \sigma_{i+1}^{y}+\sigma_{i}^{z} \sigma_{i+1}^{z}+\Delta h_{i} \sigma_{i}^{z}\right),
$$

where the $h_{i}$ are drawn from the interval $h_{i} \in[-1,1]$ and $\Delta$ denotes the disorder strength. This model is expected to undergo a localization transition at $\Delta \approx 7.5$. Moreover, we use periodic boundary conditions in order to have a meaningful definition of support for all lattice sites and denote with $\operatorname{dist}(\cdot, \cdot)$ the natural distance of two sites for a ring configuration. The Pauli operators above in the Hamiltonian denote real space spin operators acting on lattice sites $i=1, \ldots, L$ by $\sigma_{i}^{\alpha}:=\mathbb{1}_{2^{i-1}} \otimes \sigma^{\alpha} \otimes \mathbb{1}_{2^{L-i}}$, where $\sigma^{\alpha}$ for $\alpha=x, y, z$ denotes the spin- $1 / 2$ Pauli matrices and $\mathbb{1}_{n}$ the identity on $\mathbb{C}^{n}$. These operators are formulated within the standard real space basis $\left\{\left|i_{1} \ldots i_{L}\right\rangle \mid i_{j}=0,1\right\}$ which we abbreviate by $\left.\widetilde{j j}\right\rangle$ with $j=$ $1, \ldots, 2^{L}$ and $|\widetilde{j}\rangle=\left|(j-1)_{2}\right\rangle$, where $x_{2}$ denotes the binary representation of $x \in \mathbb{N}$ and we add leading 0 's on the left such that $x_{2}$ has always $L$ bits. For the following, it is useful to note that the $\sigma_{i}^{z}$ operators for $i=1, \ldots, L$ can be written as

$$
\left.\sigma_{i}^{z}=\sum_{j=1}^{2^{L}}(-1)^{\left\lfloor(j-1) / 2^{L-i}\right\rfloor} \widetilde{j}\right\rangle \widetilde{\langle j|}
$$

with $\lfloor\cdots\rfloor$ denoting the floor function.

Similarly, we introduce Pauli operators defined in energy space. Given an eigenbasis $\{|k\rangle\}$ of $H$, we specify another set of Pauli- $z$ operators through the relation

$$
\mathcal{Z}_{i}=\sum_{k=1}^{2^{L}}(-1)^{\left\lfloor(k-1) / 2^{L-i}\right\rfloor}|k\rangle\langle k| .
$$

In the infinite disorder limit $(\Delta \rightarrow \infty)$, the Hamiltonian becomes diagonal in the real space basis and hence these operators become equal to the $\left\{\sigma_{i}^{z}\right\}$ operators. For the general case with finite $\Delta$, however, the $\left\{\mathcal{Z}_{i}\right\}$ and $\left\{\sigma_{i}^{z}\right\}$ are formulated in different bases and differ from each other. Written in the given eigenbasis of $H$ it holds then that $\mathcal{Z}_{i}=\mathbb{1}_{2^{i-1}} \otimes \sigma^{z} \otimes \mathbb{1}_{2^{L-i}}$ which corresponds to a formal decomposition $\mathcal{H}=\mathbb{C}^{2^{L}} \simeq$ $\otimes_{i=1}^{L} \mathbb{C}^{2}$ that is implicitly fixed by an arbitrarily chosen order of energy eigenvalues and eigenvectors. As this is crucial for the following we emphasize that the $\mathcal{Z}_{i}=\mathbb{1}_{2^{i-1}} \otimes \sigma^{z} \otimes \mathbb{1}_{2^{L-i}}$ operators are formulated in energy space, meaning that the $\sigma^{z}$ operators here are diagonal in energy space and in principle unrelated to their real space versions. Hence, there are two decompositions of the Hilbert space into $\otimes_{i=1}^{L} \mathbb{C}^{2}$, one in real and the other in energy space. Identifying a decomposition of $\mathcal{H}$ in energy space which preserves locality in real space lies at the heart of the construction of the set of qLCOMs.

Throughout this work, the MBL constants of motion will be denoted by $\tau_{i}^{z}$. Let us summarize their desired properties:

(i) Independent conserved quantities. The $\left\{\tau_{i}^{z}\right\}$ operators must commute with $H$ and each other

$$
\left[H, \tau_{i}^{z}\right]=0 \text { and }\left[\tau_{i}^{z}, \tau_{j}^{z}\right]=0 \quad \forall i, j .
$$

(In fact, they should be functionally independent, i.e., no constant of motion can be expressed as a function of the other.)

(ii) Dressed spins. The qLCOMs are expected to have a spectrum resembling Pauli- $z$ operators, i.e., there exists a dressing unitary $U_{D}^{\dagger}$ transforming the energy to real space

$$
\tau_{i}^{z}=U_{D} \mathcal{Z}_{i} U_{D}^{\dagger}
$$

(iii) Quasilocality. For each $i$ let us denote by $S$ a "buffer" region of odd cardinality $|S|$, i.e., $S:=\{j: \operatorname{dist}(i, j) \leqslant(|S|-$ $1) / 2\}$. Then we demand that the conserved quantities must be quasilocal, meaning each $\tau_{i}^{z}$ is centered around site $i$, and its local reductions fulfill

$$
1-\frac{1}{2^{|S|+2\left|S^{C}\right|}}\left\|\operatorname{Tr}_{S^{C}}\left(\tau_{i}^{z}\right)\right\|_{2}^{2} \leqslant f(|S|),
$$

where $\operatorname{Tr}_{S^{C}}(\cdot)$ denotes the partial trace over the complement of $S$, and $f: \mathbb{N} \rightarrow \mathbb{R}^{+}$is a suitably-presumably exponentially-decaying function. Acknowledging that $\|A\|_{2}^{2}=\operatorname{Tr}\left(A^{\dagger} A\right)$, this is exactly the quantity-measuring locality discussed in Ref. [32], and it implies the locality discussed in Ref. [35]. Note that there are several possible definitions for measuring the locality of the qLCOMs. It is interesting to see that this notion of quasilocality based on the Hilbert-Schmidt norm is the sense in which it is discussed for integrable models [36-38].

Note that constructing a set of constants of motion fulfilling only properties (i) and (ii) can be easily done for systems which allow for exact diagonalization, as any set of $\left\{\mathcal{Z}_{i}\right\}$ operators constructed from any eigenbasis of $H$ will automatically satisfy (i) and (ii). Ensuring (iii), however, is nontrivial in this case and can only be obtained by choosing a correct ordering of eigenvectors of $H$ in the eigenbasis.

\section{EFFECTIVE DESCRIPTION OF LOCALIZATION}

Assuming the precise knowledge of the set of qLCOMs, it is possible to identify an effective Hamiltonian in terms of the $\left\{\tau_{i}^{z}\right\}$ operators because by properties (i) and (ii), the collection of $\left\{\tau_{i}^{z}\right\}$ and products thereof form a basis for all matrices diagonal in the chosen eigenbasis $\{|k\rangle\}$ of $H$. Given 
a set of qLCOMs the effective model [20,21] takes the form

$$
H_{\mathrm{eff}}^{\left(N_{\mathrm{eff}}\right)}=\sum_{i} \omega_{i}^{(1)} \tau_{i}^{z}+\sum_{i, j} \omega_{i, j}^{(2)} \tau_{i}^{z} \tau_{j}^{z}+\cdots,
$$

where $\cdots$ subsumes terms up to a truncation order $N_{\text {eff }}$, and $H_{\mathrm{eff}}^{(L)}=H$ if the order of the expansion reaches the system size $N_{\text {eff }}=L$. Let us introduce a subscript $\mu \in\{0,1\}^{L}$, a binary word of length $L$, which determines the position of the $\left\{\tau_{i}^{z}\right\}$ operators in the chain and define $\tau(\mu)=\prod_{i=1}^{L}\left(\tau_{i}^{z}\right)^{\mu_{i}}$. There are $2^{L}$ many of these configurations covering all possible combinations of the $\tau_{i}^{z}$ operators acting on the chain. Then, for any

$$
H=\sum_{e=1}^{2^{L}} E_{e}|e\rangle\langle e|,
$$

we may write the full expansion of Eq. (7) to order $N_{\text {eff }}=L$ as

$$
H=\sum_{\mu} \omega_{\mu} \tau(\mu)
$$

with $\omega_{\mu}=2^{-L} \operatorname{Tr}[H \tau(\mu)]$. Note that according to Eq. (5) the $\omega_{\mu}$ can be calculated in energy space via $\omega_{\mu}=$ $2^{-L} \operatorname{Tr}[\operatorname{diag}(E) \mathcal{Z}(\mu)]$ if the energies in $\operatorname{diag}(E)$ are ordered according to the ordering of the eigenbasis constructing the $\left\{\mathcal{Z}_{i}\right\}$ operators. This construction can be interpreted as a Boolean Fourier transform of the spectrum $E[39,40]$. For a specific model the weights $\left\{\omega_{\mu}\right\}$ can only be calculated that way if the different $\left\{\mathcal{Z}_{i}\right\}$ are orthogonal with respect to the HilbertSchmidt scalar product, which follows from property (ii). In the localizing case putatively realized by MBL systems, two additional restrictions are expected to hold for the couplings $\left\{\omega_{\mu}\right\}$ :

(iv) Convergence. The couplings of different orders are expected to fulfill $\omega_{\eta} \ll \omega_{\xi}$, whenever

$$
\sum_{k=1}^{L} \eta_{k}>\sum_{l=1}^{L} \xi_{l}
$$

This would imply that Eq. (7) is expected to be a good approximation of the full Hamiltonian for low $N_{\text {eff }} \ll L$.

(v) Locality. It is expected that the weights $\left\{\omega_{\mu}\right\}$ decay with the maximal distance of two $\tau_{i}^{z},\left|\omega_{\mu}\right| \leqslant g(d(\mu))$, where again $g: \mathbb{N} \rightarrow \mathbb{R}^{+}$is a suitably decaying function and $d(\mu):=$ $\max \left\{\operatorname{dist}(i, j): \mu_{i}=\mu_{j}=1\right\}$.

In later parts of this work, we explicitly construct $H_{\mathrm{eff}}$ and investigate the validity these two properties using ED.

\section{MINDSET OF THE APPROACH}

The physical intuition behind the algorithm for identifying qLCOMs proposed below is simply that real space spin operators should merely change under the infinite time average if the system is localized. Their time average will hence be diagonal in the energy eigenbasis and at the same time quasilocal in real space. We then set out to find a permutation of the eigenvectors of $H$ such that the time averages of the real space Pauli- $z$ operators best resemble Pauli- $z$ operators in energy space from which we can then construct the qLCOMs $\left\{\tau_{i}^{z}\right\}$.

The new method to construct the qLCOMs we propose here starts from the energy eigenbasis $\{|e\rangle\}$, expressed in an arbitrary but fixed ordering. For each ordering of the eigenbasis $|k\rangle=|\pi(e)\rangle$, where $\pi \in S_{2^{L}}$ is a permutation of the spectrum, we can define $\left\{\mathcal{Z}_{i}\right\}$ as above and relate them to real space $\left\{\tau_{i}^{z}\right\}$ operators as in Eq. (5). As already pointed out above, these operators by construction fulfill properties (i) and (ii). Any energy ordering $\pi \in S_{2^{L}}$ can be used to define a set of $\left\{\mathcal{Z}_{i}\right\}$, but this in general does not yield quasilocal constants of motion $\left\{\tau_{i}^{z}\right\}$ in real space. Demanding property (iii) in localized systems, the task is to identify permutations $\pi \in S_{2^{L}}$ that yield local constants of motion. However, there are $2^{L}$ ! possible permutations, hence achieving global optimality over all permutations is computationally not feasible. Having said that, we can find a solution giving rise to sufficiently local constants of motion heuristically, by exploiting the physical insight above: We order the eigenbasis such that the spectra of the dephased local magnetization operators simultaneously resemble Pauli- $z$ spectra of $\left\{\mathcal{Z}_{i}\right\}$. This turns out to be sufficient for ensuring locality of the qLCOMs $\left\{\tau_{i}^{z}\right\}$.

\section{CONSTRUCTING THE SET OF qLCOMS}

We begin by mapping each real space spin operator $\left\{\sigma_{i}^{z}\right\}$ to its infinite time average $\mathbb{E}\left(\sigma_{i}^{z}\right)=\sum_{e}\left\langle e\left|\sigma_{i}^{z}\right| e\right\rangle|e\rangle\langle e|$, where the sum goes over all eigenvectors $\{|e\rangle\}$ of $H$. This operation stems from equilibration theory $[1,41]$ and for nondegenerate Hamiltonians one has $\mathbb{E}\left(\sigma_{i}^{z}\right)=\lim _{T \rightarrow \infty}(1 / T) \int_{0}^{T} \sigma_{i}^{z}(t) d t$. This yields $L$ operators diagonal in energy space which commute among each other and with $H$ [property (i)] and are found to be quasilocal [32] [property (iii)]. However, due to the nonunitary dephasing, the spectrum of $\mathbb{E}\left(\sigma_{i}^{z}\right)$ does not satisfy (ii) and hence is only approximately Pauli-z-like. We now set out to reorder the eigenbasis $\{|e\rangle\}$ of each $\left\{\mathbb{E}\left(\sigma_{i}^{z}\right)\right\}$ with a permutation $\pi \in S_{2^{L}}$ such that $\left\{\mathbb{E}\left(\sigma_{i}^{z}\right)\right\}$ written in the reordered basis $\{|k=\pi(e)\rangle\}$ best resemble $\left\{\mathcal{Z}_{i}\right\}$ in the sense that the entrywise difference between each $\mathbb{E}\left(\sigma_{i}^{z}\right)$ and $\mathcal{Z}_{i}$ is small. We construct the reordered basis $\{|k\rangle\}$ using a heuristic scheme in multiple steps by considering each $\mathbb{E}\left(\sigma_{i}^{z}\right)$ successively.

The structure of all $\left\{\mathcal{Z}_{i}\right\}$ is by construction known (see for instance the black dashed line in Fig. 1 which indicates the diagonal of $\mathcal{Z}_{1}, \mathcal{Z}_{2}, \mathcal{Z}_{3}$, and $\mathcal{Z}_{4}$ in the different panels from top to bottom). For each $\mathbb{E}\left(\sigma_{i}^{z}\right)$, we then identify a permutation by which $\mathbb{E}\left(\sigma_{i}^{z}\right)$ best approximates $\mathcal{Z}_{i}$ without altering the result identified for previous $\mathbb{E}\left(\sigma_{j}^{z}\right)$ with $j<i$ by sorting the eigenvectors only in the degenerate subspaces of $\mathcal{Z}_{i-1}$ according to the size of the eigenvalues of $\mathbb{E}\left(\sigma_{i}^{z}\right)$ and not allowing for a mixing between those subspaces. To illustrate the concept, consider the operator $\mathcal{Z}_{1}=\sigma^{z} \otimes \mathbb{1}_{2^{L-1}}$ in the energy eigenbasis. It is diagonal in the desired basis $\{|k\rangle\}$ and takes the form $\mathcal{Z}_{1}=\mathbb{1}_{2^{L-1}} \oplus-\mathbb{1}_{2^{L-1}}$. Hence, the entrywise closest permutation of $\mathbb{E}\left(\sigma_{1}^{z}\right)$ is simply sorting its spectrum by size (cf. Fig. 1, first row). Note that this choice is highly nonunique, as it allows for an arbitrary order inside the two degenerate sectors. We will use this ambiguity to optimize the remaining qLCOMs. Next, $\mathcal{Z}_{2}$ has the form $\mathcal{Z}_{2}=$ $\mathbb{1}_{2^{L-2}} \oplus-\mathbb{1}_{2^{L-2}} \oplus \mathbb{1}_{2^{L-2}} \oplus-\mathbb{1}_{2^{L-2}}$. The infinite time average $\mathbb{E}\left(\sigma_{2}^{z}\right)$ gives us a new spectrum to optimize. We then exploit the fact that in the degenerate sectors of $\mathcal{Z}_{1}$ our ordering is at the moment arbitrary, i.e., not fixed by $\mathbb{E}\left(\sigma_{1}^{z}\right)$. In the second step, we therefore sort each of the two sectors by size of the spectrum of $\mathbb{E}\left(\sigma_{2}^{z}\right)$ (cf. Fig. 1, second row). It is important to note that we 


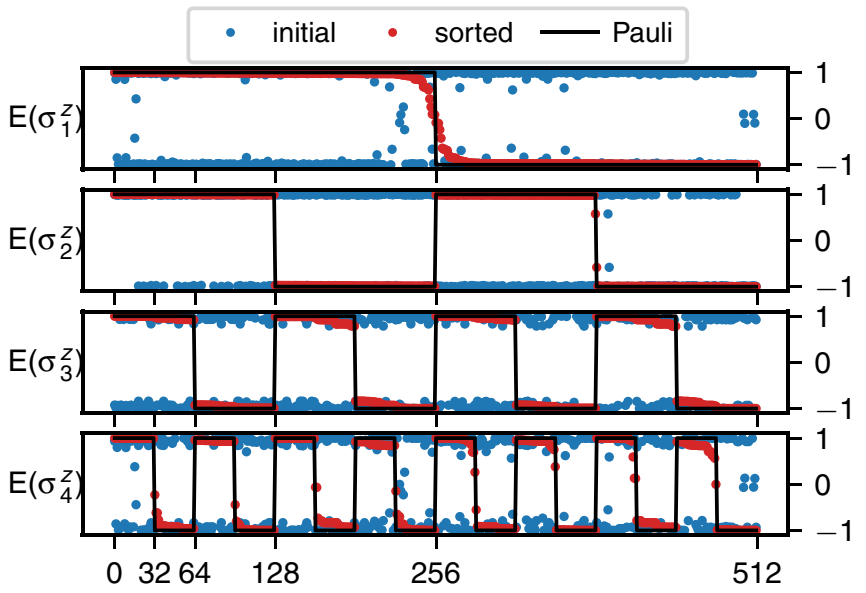

FIG. 1. Energy eigenbasis obtained from ED calculations is defined up to a permutation basis which may obscure the physical content available in the infinite time average of local spin operators. Here we show data for a specific disorder realization with $\Delta=20$ on $L=9$ sites. The plots show the size of the eigenvalues of the infinite time-averaged real space Pauli operators $\mathbb{E}\left(\sigma_{i}^{z}\right)$. We illustrate our procedure of permuting the eigenvalues of the set $\mathbb{E}\left(\sigma_{i}^{z}\right)$ to obtain a particular diagonalization unitary $U_{D}$ that ensures locality properties of Pauli- $z$ operators $\mathcal{Z}_{i}$ when rotated into the real space basis $\tau_{i}^{z}$. The difference of $\mathbb{E}\left(\sigma_{i}^{z}\right)$ to $\mathcal{Z}_{i}$ comes from the discrepancy of the spectra due to the dephasing.

must not swap entries from the different sectors with one another as this would spoil the formerly established permutations. This procedure is iterated for the remaining $\mathbb{E}\left(\sigma_{i}^{z}\right)$ as shown in Fig. 1. Ultimately, for fixing the last permutation $\pi_{L} \in S_{2^{L}}$, we only have the freedom to sort in $2^{L} / 2$ many blocks of size 2 , namely, to perform swaps for neighboring eigenvectors only. As a result we find the final ordering $\pi=\pi_{L} \circ \cdots \circ \pi_{1}$ and we collect the resulting basis to the unitary $U_{D}$ of Eq. (5) that can be used to represent the qLCOMs in real space. To be precise, we now use the obtained $U_{D}$ to transform the $\left\{\mathcal{Z}_{i}\right\}$ which by construction fulfill properties (i) and (ii) into real space. The following pseudocode describes a possible way to implement this procedure numerically. We use a notation close to PYTHON and denote, for instance, for a list $l$ of numbers $1, \ldots, N$ in an arbitrary order, a vector $v \in \mathbb{C}^{N}$ and a matrix $U \in \mathbb{C}^{N \times N}$ by $v[l]$ and $U[:, l]$ the vector and matrix for which the elements of the vector and columns of the matrix are reordered according to $l$, i.e., $v[l]_{i}=v_{l_{i}}$ and $U[:, l]_{i, j}=U_{i, l_{j}}$. Similarly, we denote for $v \in \mathbb{C}^{N}$ and $1 \leqslant n<m \leqslant N$ by $v[n: m]$ the vector $v[n: m] \in$ $\mathbb{C}^{m-n}$ with entries $v[n: m]_{i}=v_{n+i-1}$.

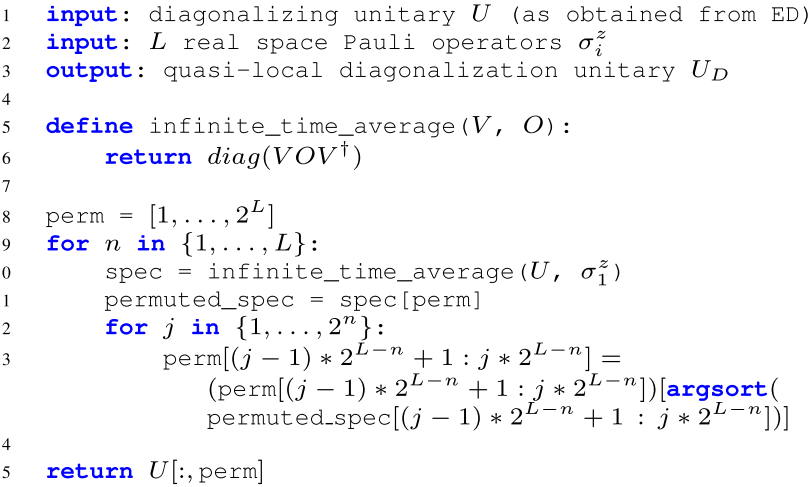

\section{NUMERICAL RESULTS}

We now examine the properties of the qLCOMs constructed according to the above scheme. First, we make sure that the obtained operators are indeed quasilocal and hence fulfill property (iii). We find that the qLCOMs constructed with our algorithm are local to a few sites only at high enough disorder, an observation which reproduces the theoretical predictions. In Fig. 2 we plot the support of the first, last, and averaged over all constructed $\left\{\tau_{i}^{z}\right\}$ of the 13-site lattice as a function of disorder strength $\Delta$ averaged over 300 realizations. The quantifier for the support is the truncation error to a subsystem $S$ in 2-norm defined as $1-\left\|\operatorname{Tr}_{S^{C}}\left(\tau_{i}^{z}\right)\right\|_{2}^{2} /\left(2^{|S|+2\left|S^{C}\right|}\right)$. If the value is close to unity, the spectrum of the operator deviates

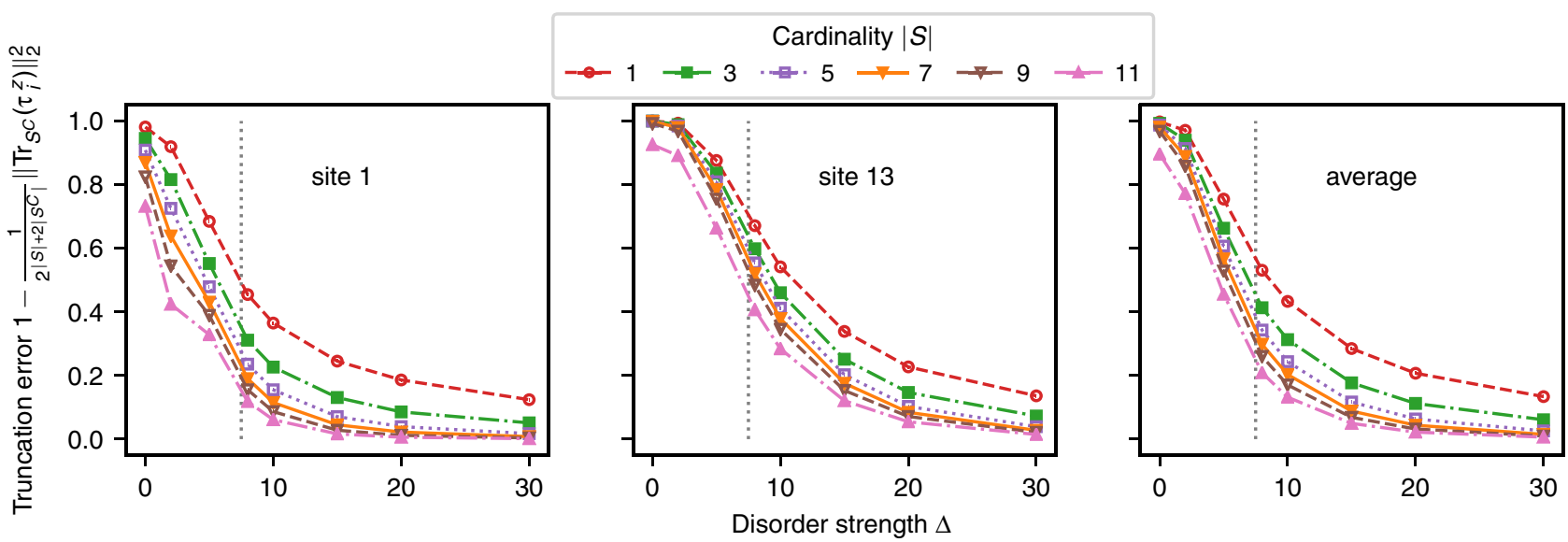

FIG. 2. Support of the first (left panel) and the last (center) qLCOMs as well as the averaged support of all constructed qLCOMs (right panel) of the disorder Heisenberg chain over the disorder strength $\Delta$. As a measure of support, we use the truncation error $1-\operatorname{Tr}_{S}\left(\tau_{i}^{z}\right) \|_{2}^{2} /\left(2^{|S|+2\left|S^{C}\right|}\right)$ for buffer regions $S$ of increasing cardinality $|S|$. Error bars show statistical deviations over 300 realizations. We use the Heisenberg Hamiltonian on $L=13$ with periodic boundary conditions. The dashed lines at $\Delta=7.5$ are a guide to the eye indicating the region of the expected phase transition. 


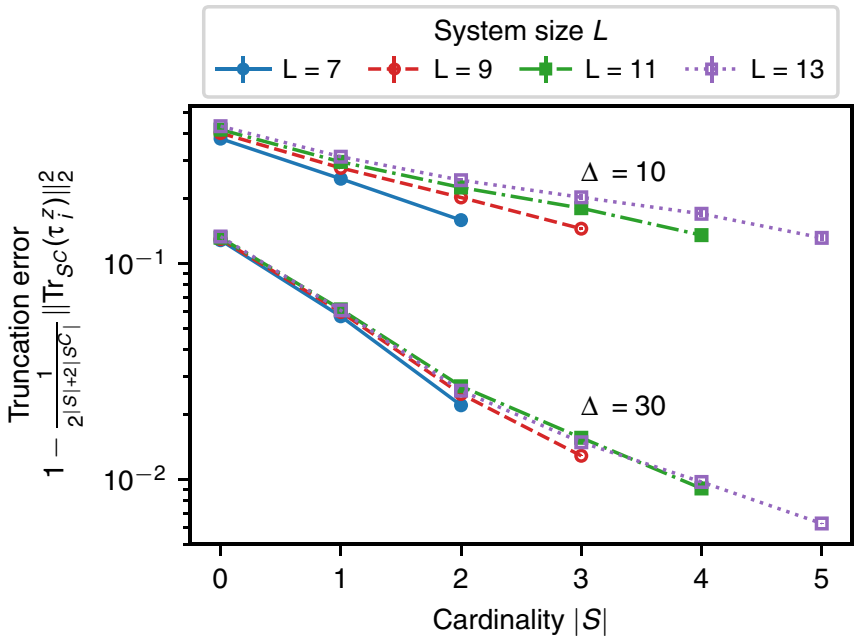

FIG. 3. Average truncation error with random disorder for $L \in$ $\{7,9,11,13\}$ with $\{1000,1000,1000,300\}$ realizations. We use the Heisenberg Hamiltonian with the disorder strength $\Delta \in\{10,30\}$. Moreover we employ periodic boundary conditions. The plot shows the average truncation error $1-\left\|\operatorname{Tr}_{S} C\left(\tau_{i}^{z}\right)\right\|_{2}^{2} /\left(2^{|S|+2\left|S^{C}\right|}\right)$ of the $\mathrm{qL}$ COMs when truncated onto a "buffer" region of off cardinality $|S|$ averaged over all qLCOMs. The plot is on a log scale. Lines are guide to the eye. Error bars show statistical deviations.

strongly from the Pauli- $z$ spectrum. If the value is zero, the operator is in this sense well characterized by its reduction to the subsystem $S$. We find that increasing disorder localizes the obtained operators. Additionally, one observes a crossover in the region of the proposed phase transition. It can furthermore be seen that despite the recursive nature of our approach, which allows more variational freedom in the first initial qLCOMs, there is only a small systematic error between the first and last qLCOM, and all qLCOMs are well localized for $\Delta$ large enough. A finite size scaling is discussed in the following indicating that while our method works for the system sizes considered, it suggests inconclusive results for the locality of the operators for larger systems. Figure 4 displays the averaged decay of the qLCOMs and shows that in the localized phase $1-\left\|\operatorname{Tr}_{S^{c}}\left(\tau_{i}^{z}\right)\right\|_{2}^{2} /\left(2^{|S|+2\left|S^{C}\right|}\right)$ decays exponentially showing that the $\left\{\tau_{i}^{z}\right\}$ are local up to exponential tails. Here we average both over realizations and qLCOMs per realization. Additionally one observes a stronger decay for larger disorder. This scaling with the disorder strength is very much expected and consistent with theoretical predictions. Next, we study the finite size dependence of the locality results. Fig. 3 shows the system size dependence of the truncation error $1-\left\|\operatorname{Tr}_{S^{C}}\left(\tau_{i}^{z}\right)\right\|_{2}^{2} /\left(2^{|S|+2\left|S^{C}\right|}\right)$ of the qLCOMs for moderate $(\Delta=10)$ and strong $(\Delta=30)$ disorder. The qualitative behavior between the disorder strengths is consistent with Fig. 4. When considering increasing system sizes, we observe that the decay slows down. Nevertheless, we see that for all system sizes we obtain a strong decay with the distance. For the system sizes accessible, we find a still sufficient decay to call the obtained qLCOMs quasilocal. However, it seems hard to predict the trend for larger systems based on the given data. Let us now turn to insights about the transition between the MBL and the ergodic phase.

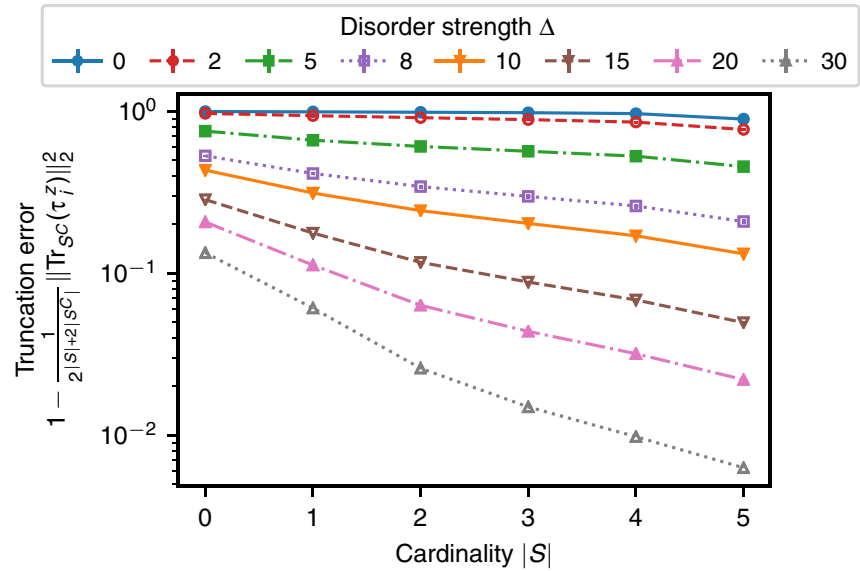

FIG. 4. Decay of the average truncation error over all constants of motion displayed in Fig. 3 on a log scale for different disorder strengths. The error bars indicate the standard deviation of the average and lines are a guide to the eye.

An interesting open question is how precisely the picture of the qLCOMs breaks down once the transition toward the ergodic phase is being approached. Intuitively, one expects a broadening of the qLCOMs upon delocalizing, which ultimately leads to completely nonlocal constants of motion. Here, we set out to observe this transition in the locality of the calculated qLCOMs. The measure we employ is the the cardinality of the minimal buffer region $S$ (see above) needed to support as much as a threshold $\alpha$ of the weight of the operator. We again work with the squared two-norm of the reduced operator as a quantifier of support. We show the results in Fig. 5 for different thresholds $\alpha \in\{0.5,0.6,0.7,0.8\}$. While the resulting curve clearly depends on the chosen threshold, a transition between a phase, where the operator is supported

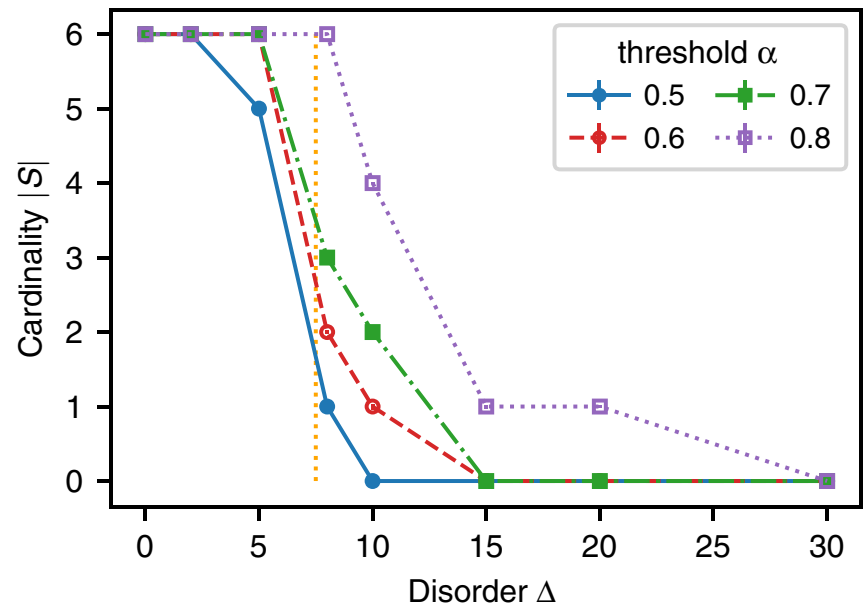

FIG. 5. Cardinality of the minimal buffer region for threshold values $\alpha \in\{0.5,0.6,0.7,0.8\}$. Values are obtained for the disordered Heisenberg model on $L=13$ with periodic boundary conditions. Each data point comprises 300 realizations averaged over all qLCOMs. Lines are guide to the eye. Error bars show statistical deviations. The orange dotted line indicates the expected transition at $\Delta=7.5$. 


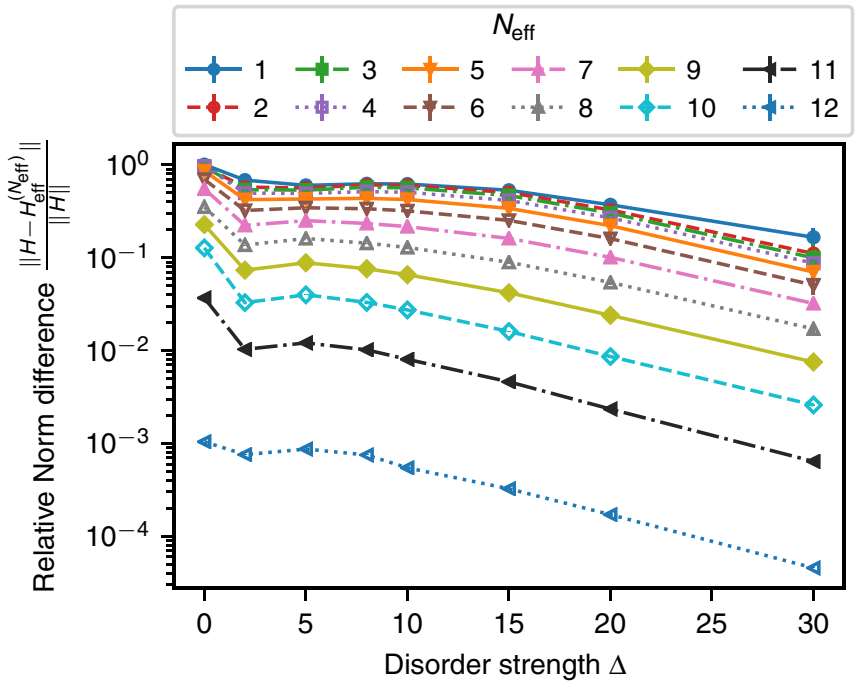

FIG. 6. Relative norm difference between effective model and actual Hamiltonian $\left\|H-H_{\text {eff }}^{\left(N_{\text {eff }}\right)}\right\| /\|H\|$ on $L=13$ with random disorder on a $\log$ scale. Different colors indicate the order of the approximation $N_{\text {eff }}$. Error bars show statistical deviations. The average is performed over 300 realizations. Lines are a guide to the eye.

on the full system for low disorder and on a single site for high disorder can clearly be observed. To precisely identify the phase boundary is a challenge for all known methods, and this one is no exception. While the measure we propose here may not give a reliable quantitative estimate of the transition, it nevertheless provides a clear qualitative one. Furthermore, it strengthens the intuition of the nature of the phase transition, giving rise to a broadening of qLCOMs.

Using the constructed qLCOMs, we now turn to the effective model and investigate its properties in detail. We would like to point out that this is only possible since our set of qLCOMs fulfills properties (i)-(iii) exactly and not only approximately and hence offers the algebraic structure necessary to exactly construct the effective description. We compute the weights $\omega_{\mu}$ in energy space as explained before using the orthogonality of the $\left\{\tau_{i}^{z}\right\}$ operators and show their decay in Fig. 7. While the $\omega_{\mu}$ decay with increasing spatial extension $d(\cdot)$, there is no apparent inter-order decay. Moreover, there is an apparent saturation for higher orders. This allows two explanations: Either the qLCOMs can be further optimized to fit the expectations of the effective description better or the Heisenberg model cannot be mapped to the effective model with strongly decaying couplings. A possible measure of where to set an effective cutoff $N_{\text {eff }}$ is the operator norm distance of the Hamiltonian $H$ and its effective description $H_{\text {eff }}^{\left(N_{\text {eff }}\right)}$. Figure 6 shows the scaling of $\left\|H-H_{\text {eff }}^{\left(N_{\text {eff }}\right)}\right\| /\|H\|$ in the dependence on the disorder strength $\Delta$ with $N_{\text {eff }}$ as a parameter. Here, we observe that indeed all orders do decay with an exponential trend for larger disorder. However, to get the norm error small, a rather large $N_{\text {eff }}$ has to be chosen. This seems to put the validity of the effective description as a full solution in question. However, note that we cannot rule out that qLCOMs can be found that allow a better effective model as also stated previously. For a brute force approach, $2^{L}$ ! many

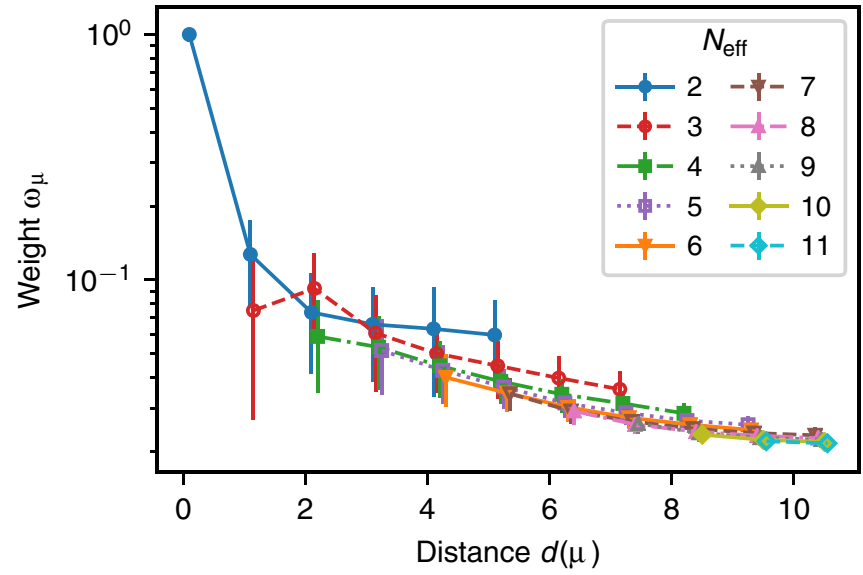

FIG. 7. Average coupling strength $\omega_{\mu}$ on $L=13$ with random disorder of strength $\Delta=20$ on a log scale. Different colors indicate the order of the approximation $N_{\text {eff }}$. Error bars show statistical deviations of the average over 300 realizations and per realization over all operators with support of extension $d(\mu)$. Lines are a guide to the eye.

configurations have to be checked, which quickly out scales any computational resources. Hence it will be necessary to work with a heuristic such as the one presented in this work. Devising new heuristics which can better fit the effective model will be part of future research.

However, imposing such strict global constraints as done by the operator norm difference of the exact and effective Hamiltonian may not be required to recover the essential physical behavior of the system. Hence we will investigate the predictions of the effective model on a local scale in the following.

\section{OBTAINING LOCAL DYNAMICS FROM THE EFFECTIVE DESCRIPTION}

To provide more substance to this discussion, we investigate the nonequilibrium quench dynamics of local observables akin to recent experiments [8]. We compare the dynamical evolution of the imbalance

$$
\mathcal{I}=\frac{1}{L} \sum_{i}(-1)^{i} \sigma_{i}^{z},
$$

where the initial state is a real space Neel state vector $|1,0, \ldots, 1,0,1\rangle$ for the Heisenberg Hamiltonian and the effective description truncated to order $N_{\text {eff }}=4$. In Fig. 8 we pick three realizations based on the norm difference $\left\|H-H_{\mathrm{eff}}^{(4)}\right\|$, namely, the worst, intermediately good, and best one. We find quantitative agreement of the dynamical evolution when the low-order effective description is close in operator norm to the true Hamiltonian; however, there may exist realizations where the phenomenological model would demand many higherorder terms as seen for the bad realization (red in Fig. 8). Notably, the effective description fails to reproduce quantitatively fast oscillations of the imbalance, but the qualitative behavior, e.g., the average imbalance value, is still captured. For realizations that work intermediately well, the quantitative agreement is lost over time. 


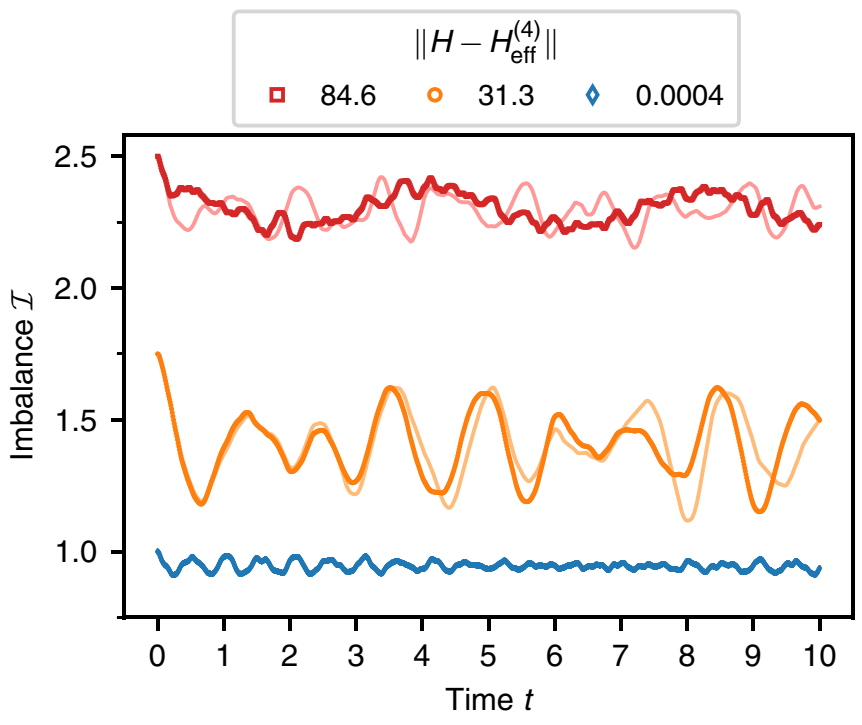

FIG. 8. Dynamics of the imbalance in the Neel state of $L=13$ spins with periodic boundary conditions with disorder strength $\Delta=$ 20. The plot shows a comparison of the exact dynamics (solid lines) in three realizations (picked by norm difference $\left\|H-H_{\text {eff }}^{(4)}\right\|$ of 30 realizations) with the evolution generated by the truncated effective Hamiltonian $H_{\text {eff }}^{(4)}$ (symbols).

\section{SUMMARY AND OUTLOOK}

In this work, we have proposed an algorithm for numerically constructing exact constants of motion in the localized phase of models exhibiting MBL, with an emphasis on the random field Heisenberg chain. In contrast to previous attempts of numerically tackling MBL systems, we have put strong emphasis on exactly fulfilling all desired commutation relations as well as obtaining a Pauli- $z$ spectrum of the constructed operators. Based on this paradigm, our algorithm finds operators which furthermore act quasilocally in real space in the localized regime. Equipped with a full set of exact qLCOMs, we are able to explicitly calculate the effective description of localized systems to all orders. It is the hope that this tool to construct exact effective Hamiltonians can help to satisfactorily explore the rich phenomenology of many-body localized systems. For future work, it appears a natural question to investigate whether the equilibrium state of MBL systems can as anticipated be described by generalized Gibbs ensembles featuring the qLCOM. As MBL systems can be tuned between "ergodicity" and "integrability," progress in this direction may also shed light on thermalization in more general models. Moreover, we aim at elevating the present method to a tensor network consisting of many subsystems, iterating steps, to give rise to a two-layer quantum cellular automaton, reminiscent of the tensor network of Ref. [19]. It is the hope that equipped with exact constants of motion and effective models, the present work can contribute to resolving the remaining puzzles on many-body localization in one spatial dimension.

Note added. Recently we became aware of the independent similar work presented in Refs. [42-44].

\section{ACKNOWLEDGMENTS}

We thank J. Behrmann, D. Litinski, and H. Wilming for helpful comments. This work has been supported by the ERC (TAQ), the DFG (CRC 183, A2, EI 519/7-1, and EI 519/14-1), the Templeton Foundation, and the EC (AQuS).
[1] J. Eisert, M. Friesdorf, and C. Gogolin, Nat. Phys. 11, 124 (2015).

[2] A. Polkovnikov, K. Sengupta, A. Silva, and M. Vengalattore, Rev. Mod. Phys. 83, 863 (2011).

[3] C. Gogolin and J. Eisert, Rep. Prog. Phys. 79, 56001 (2016).

[4] P. W. Anderson, Phys. Rev. 109, 1492 (1958).

[5] D. M. Basko, I. L. Aleiner, and B. L. Altshuler, Ann. Phys. (NY) 321, 1126 (2006).

[6] V. Oganesyan and D. A. Huse, Phys. Rev. B 75, 155111 (2007).

[7] D. J. Luitz, N. Laflorencie, and F. Alet, Phys. Rev. B 91, 081103 (2015).

[8] M. Schreiber, S. S. Hodgman, P. Bordia, H. P. Lüschen, M. H. Fischer, R. Vosk, E. Altman, U. Schneider, and I. Bloch, Science 349, 842 (2015).

[9] J.-Y. Choi, S. Hild, J. Zeiher, P. Schauß, A. Rubio-Abadal, T. Yefsah, V. Khemani, D. A. Huse, I. Bloch, and C. Gross, Science 352, 1547 (2016).

[10] M.Znidaric, T. Prosen, and P. Prelovsek, Phys. Rev. B 77, 064426 (2008).

[11] J. H. Bardarson, F. Pollmann, and J. E. Moore, Phys. Rev. Lett. 109, 017202 (2012).

[12] B. Bauer and C. Nayak, J. Stat. Mech. (2013) P09005.

[13] J. Eisert, M. Cramer, and M. B. Plenio, Rev. Mod. Phys. 82, 277 (2010).

[14] M. Friesdorf, A. H. Werner, W. Brown, V. B. Scholz, and J. Eisert, Phys. Rev. Lett. 114, 170505 (2015).
[15] X. Yu, D. Pekker, and B. K. Clark, Phys. Rev. Lett. 118, 017201 (2017).

[16] V. Khemani, F. Pollmann, and S. L. Sondhi, Phys. Rev. Lett. 116, 247204 (2016).

[17] F. Pollmann, V. Khemani, J. I. Cirac, and S. L. Sondhi, Phys. Rev. B 94, 041116 (2016).

[18] D. M. Kennes and C. Karrasch, Phys. Rev. B 93, 245129 (2016).

[19] T. B. Wahl, A. Pal, and S. H. Simon, Phys. Rev. X 7, 021018 (2017).

[20] D. A. Huse, R. Nandkishore, and V. Oganesyan, Phys. Rev. B 90, 174202 (2014).

[21] M. Serbyn, Z. Papić, and D. A. Abanin, Phys. Rev. Lett. 111, 127201 (2013).

[22] M. Serbyn, Z. Papić, and D. A. Abanin, Phys. Rev. Lett. 110, 260601 (2013).

[23] M. Friesdorf, A. H. Werner, M. Goihl, J. Eisert, and W. Brown, New J. Phys. 17, 113054 (2015).

[24] J. Z. Imbrie, J. Stat. Phys. 163, 998 (2016).

[25] R. Vosk and E. Altman, Phys. Rev. Lett. 110, 067204 (2013).

[26] L. Rademaker and M. Ortuño, Phys. Rev. Lett. 116, 010404 (2016).

[27] L. Rademaker, M. Ortuno, and A. M. Somoza, Ann. Phys. (NY) 529, 1600322 (2017).

[28] C. Monthus, J. Phys. A 49, 305002 (2016). 
[29] D. Pekker, B. K. Clark, V. Oganesyan, and G. Refael, Phys. Rev. Lett. 119, 075701 (2017).

[30] W. De Roeck, F. Huveneers, M. Müller, and M. Schiulaz, Phys. Rev. B 93, 014203 (2016).

[31] D. J. Luitz, F. Huveneers, and W. De Roeck, Phys. Rev. Lett. 119, 150602 (2017).

[32] A. Chandran, I. H. Kim, G. Vidal, and D. A. Abanin, Phys. Rev. B 91, 085425 (2015).

[33] T. E. O’Brien, D. A. Abanin, G. Vidal, and Z. Papić, Phys. Rev. B 94, 144208 (2016).

[34] R.-Q. He and Z.-Y. Lu, Chin. Phys. Lett. 35, 027101 (2018).

[35] J. Z. Imbrie, V. Ros, and A. Scardicchio, Ann. Phys. (NY) 529, 1600278 (2017).

[36] E. Ilievski, M. Medenjak, T. Prosen, and L. Zadnik, J. Stat. Mech. (2016) 064008.
[37] M. Mierzejewski, P. Prelovšek, and T. Prosen, Phys. Rev. Lett. 114, 140601 (2015).

[38] M. Mierzejewski, T. Prosen, and P. Prelovšek, Phys. Rev. B 92, 195121 (2015).

[39] A. Montanaro and T. J. Osborne, Chicago J. Theoret. Comput. Sci. 1, 1 (2010).

[40] R. De Wolf, Th. Comp. 1, 1 (2008).

[41] N. Linden, S. Popescu, A. J. Short, and A. Winter, Phys. Rev. E 79, 061103 (2009)

[42] A. K. Kulshreshtha, A. Pal, T. B. Wahl, and S. H. Simon, arXiv:1707.05362.

[43] S. J. Thomson and M. Schiró, Phys. Rev. B 97, 060201 (2018).

[44] M. Mierzejewski, M. Kozarzewski, and P. Prelovsek, Phys. Rev. B 97, 064204 (2018). 